\title{
Abnormalities of heart rate turbulence and heart rate variability as indicators of increased cardiovascular risk in patients with systemic sclerosis
}

\author{
Adam R. Poliwczak ${ }^{1}$, Elżbieta Waszczykowska², Bożena Dziankowska-Bartkowiak², Katarzyna Dworniak-Pryca ${ }^{3}$ \\ 1Department of Human Physiology, Medical University of Lodz, Lodz, Poland \\ 2Department of Dermatology and Venereology, Medical University of Lodz, Lodz, Poland \\ ${ }^{3}$ Department of Internal Medicine and Geriatrics, Regional Specialized Hospital, Zgierz, Poland \\ Adv Dermatol Allergol 2019; XXXVI (6): 707-713 \\ DOI: https://doi.org/10.5114/ada.2019.83134
}

\begin{abstract}
Introduction: Systemic sclerosis (SSc) is a connective tissue disease manifested by progressive fibrosis of many internal organs including the cardiovascular system and development of autonomic disorders with sympathetic predominance. These abnormalities can increase cardiovascular mortality.

Aim: To evaluate heart rate turbulence (HRT) and variability (HRV) parameters (indicator of autonomic imbalance) obtained from 24-hour ECG Holter monitoring, as predictors of the increased cardiovascular risk in patients with scleroderma.

Material and methods: Thirty-two scleroderma patients and 30 healthy people were included. After clinical examination, ECG, routine laboratory tests and echocardiography, participants performed 24-hour Holter-ECG at home. For HRT assessment, turbulence onset (To) and turbulence slope $(T s)$ parameters were used. Both time and frequency domain analysis of HRV was used. The HRV circadian rhythm was also evaluated.

Results: Time domain: SDNN, SDNN-ix, SDANN and frequency domain: LF, VLF, ULF, NHF, NLF, parameters were lower, while p50NN was higher in SSc as compared to the control group. There was also a loss of the circadian rhythm for r-MSSD and p50NN present in the control group. Abnormal HRT parameters To and/or Ts occurred in the SSc group only. The median value of $T o=-1.24 \%$ and $T s=11.13 \mathrm{~ms} / \mathrm{RR}$ did not differ significantly as compared to the control group.

Conclusions: The study confirmed the presence of HRV disturbances, including HRV circadian rhythm, as it may seem at an early stage of SSc. The HRT disorders may be characterized by the increasing changes with advancing disease. This indicates the presence of autonomic imbalance and an increased cardiovascular risk.
\end{abstract}

Key words: systemic sclerosis, autonomic disorders, heart rate variability, heart rate turbulence, cardiovascular risk, circadian rhythm.

\section{Introduction}

Systemic sclerosis (SSc) is a connective tissue disease manifested by involvement not only of the skin and subcutaneous tissue, but also many internal organs. It is characterized by progressive fibrosis and is associated with a significantly higher mortality compared with the general population [1-4]. The increased mortality is mainly due to involvement of the internal organs and the presence of specific autoantibodies [5]. The mortality rate in patients with SSc is from 1.5 to 7.2 times higher than that of the general population. Cardiac involvement appeared to increase the death rate by 2.8 times with no difference between disseminated and localized forms [5]. It is postulated that also microcirculation abnormalities, endothelial lesions and microvascular "Raynaud's phenomenon" are involved in the intensification of changes within the myocardium [1, 6]. Fibrosis and its maldistribution result in a significant electrical heterogeneity of the heart and the increased risk of severe arrhythmia, including ventricle life-threatening arrhythmia [7]. It is postulated that the microvascular sympathetic-parasympathetic imbalance potentiates endothelial damage and accelerates the development of myocardial fibrosis [8-11].

Address for correspondence: Adam R. Poliwczak MD, PhD, Department of Human Physiology, Medical University of Lodz, 1 Haller Square, 90-647 Lodz, Poland, phone: +48 602517 311, e-mail: polczak@mp.pl; adam.poliwczak@umed.lodz.pl Received: 16.03.2018, accepted: 15.07.2018. 
One of the acknowledged method of assessing the cardiovascular risk and efficiency of the autonomic nervous system is the measurement of heart rate variability (HRV) and heart rate turbulence (HRT) [12, 13]. Typically 24-hour Holter ECG recording is used. The assessment takes into account many parameters of HRV and HRT. Some data show a heightened risk of dangerous arrhythmias and increase in mortality in case of abnormal HRV and HRT values in various disease states, including heart failure, after myocardial infarction, cirrhotic cardiomyopathy and others [14-16].

The phenomenon of heart rate turbulence describes the short-term heart rate variability occurring during the sinus rhythm that appears after a premature or paced ventricular contraction. This leads to an initial acceleration (early acceleration) and then deceleration (late deceleration) of the heart rate. Measurement of these two consecutive phases is obtained through To (turbulence onset) and Ts (turbulence slope) parameters. Normal values of these parameters are To $<0 \%$ and Ts $>2.5 \mathrm{~ms}$ [12]. There are divergent data regarding differences in HRV and HRT parameters comparing SSc patients with healthy people [8-11, 17, 18].

\section{Aim}

The aim of this study was to evaluate heart rate turbulence and heart rate variability parameters (indicator of autonomic imbalance) obtained from 24-hour ECG Holter monitoring, as predictors of the increased cardiovascular risk in patients with scleroderma.

\section{Material and methods}

The initial qualification for the study included 54 consecutive ambulatory patients. Scleroderma was diagnosed in accordance with the criteria of the American College of Rheumatology [19]. All patients underwent a basic clinical examination, had the ECG, routine laboratory tests and echocardiography performed. In the past 12 months all the patients underwent a chest radiography or high-resolution computed tomography (HRCT) and spirometry. Furthermore, each participant had plasma autoantibodies typically present in SSc. Exclusion criteria included myocardial infarction, liver cirrhosis, heart failure class II and above, according to the NYHA classification, other than sinus heart rhythm assessed by ECG, uncontrolled hypertension, renal insufficiency (GFR $<30 \mathrm{ml} / \mathrm{min} / 1.72 \mathrm{~m}^{2}$ ), diabetes, autoimmune diseases other than scleroderma, current smoking and drinking alcohol. The study included only patients without significant changes in laboratory tests and echocardiography, without pulmonary hypertension (HP) and history of significant disorders, cardiovascular disorders, inflammatory diseases and cancer (patients with the history of successful treatment of cancer longer than 5 years were considered healthy). According to the above criteria, 32 people were qualified for the study.

The control group comprised 30 people selected according to sex and age, not treated for autoimmune diseases as these constitute exclusion criteria. People included in the study underwent 24-hour Holter ECG at home.

Because of the fact that the study included one male only, statistical analysis was performed only for the group of 31 women.

Written informed consent was obtained from all the participants.

\section{4-hour ECG Holter monitoring and further analysis}

All participants underwent the 24-hour ECG recording. This study was carried out using the Aspect 702 recorder by Aspel Zabierzów, Poland. The quality of obtained data was assessed and verified by a cardiologist experienced in non-invasive electrocardiology. Records with sufficient quality were subsequently subjected to automatic computer analysis - Holcard analysis system by Aspel Zabierzów, Poland. This was followed by an automatic detection of QRS complexes, the parameters of heart rate turbulence and analysis of time and frequency domain heart rate variability.

The study used two most common HRT indices - To and Ts $[20,21]$. Their analysis and calculation were performed automatically by applications, which were a part of a commercial Holter analysis system.

For the evaluation of HRV, the Fast Fourier Transformation test was used. The assessment was performed for the entire 24-hour observation period. For further comparisons, both time and frequency domain analyses of HRV were used. The study used the typical time domain parameters - the average RR intervals of sinus rhythm, SDNN, SDANN, SDNNI - SDNN index, r-MSSD, p50NN, Trl - HRV triangular index, TINN - the triangular interpolation of NN interval histogram and the following frequency domain parameters - total power of frequency domain (TP), high frequency domain (HF), low frequency domain (LF), very low frequency domain (VLF), ultra low frequency domain (ULF), normalized HF power (NHF), normalized LF power (NLF) and LF/HF ratio.

The obtained parameters were analysed separately for the whole day and for the morning activity hours between 8:00 and 12:00 and night resting hours - between 24:00 and 4:00.

\section{Ethics}

The study was performed in agreement with the ethical guidelines of the 1975 Declaration of Helsinki and was approved by the Bioethics Committee of the Medical University of Lodz No. RNN/591/09/KB. 


\section{Statistical analysis}

Statistical analysis was performed using Statistica $8 \mathrm{PL}$ (StatSoft Inc.). The distribution of the population was verified by the Shapiro-Wilk test. Continuous variables usually differed from normal distribution. To conclude, for the significance of differences between the groups, ANOVA, Kruskal-Wallis rank test, Mann-Whitney and Wilcoxon nonparametric tests were used. For the data of close to normal distribution, Student's t-test was used. Statistical significance was indicated for $p<0.05$. As in some other works, frequency domain parameters were also presented in the form of a natural logarithm. Results were presented as mean and standard deviation and as median, and minimum and maximum values. The existence of any correlation between the parameters was tested using Spearman rank correlation test.

\section{Results}

Study groups did not differ significantly in terms of basic anthropometric parameters. Patients with scleroderma were characterized only by a higher minimum heart rate, compared with the control group (52.42/ $\min$ vs. $47.73 / \mathrm{min} ; p<0.005)$. These data are presented in Table 1. The median duration of the disease was $5.5(0.67-20.0)$ years. $73.3 \%$ of the women were postmenopausal and in the control group they accounted for $57.0 \%$ ( $p<0.05)$. In the group of SSc, ANCA occurred in $16.6 \%$, Acl-70 in 50\% and anti-Ro-52 in 20\%. Nineteen persons had the ISSC and $12 \mathrm{dSSC}$ form. Bearing in mind the opinion presented in the current literature that there are no significant differences in the studied HRT and HRV parameters, the analysis was conducted jointly for both forms [8, 18, 22]. There were no statistically significant differences in the echocardiographic parameters of the left ventricular function, including ejection fraction - EF $=66.6 \pm 4.7 \%$ vs. $68.6 \pm 4.0 \%$ for SSc and control groups, besides a significantly more frequent impaired diastolic

Table 1. Comparison of basic parameters of the tested groups

\begin{tabular}{lccc}
\hline Parameter & SCL $(n=31)$ & $\begin{array}{c}\text { Control } \\
(n=30)\end{array}$ & $P$-value \\
\hline Age & $53.94 \pm 10.64$ & $53.53 \pm 10.53$ & 0.863 \\
\hline Weight & $66.07 \pm 13.40$ & $68.12 \pm 10.73$ & 0.360 \\
\hline Height & $160.74 \pm 8.51$ & $162.87 \pm 6.18$ & 0.155 \\
\hline BMl & $25.61 \pm 5.22$ & $25.73 \pm 4.37$ & 0.735 \\
\hline Waist & $84.15 \pm 12.68$ & $84.22 \pm 9.63$ & 0.795 \\
\hline Max HR [beats/min] & $125.94 \pm 10.20$ & $123.00 \pm 7.99$ & 0.983 \\
\hline Min HR [beats/min] & $52.42 \pm 6.22$ & $47.73 \pm 5.33$ & $<0.005$ \\
\hline Mean HR [beats/min] & $83.65 \pm 7.19$ & $80.63 \pm 6.76$ & 0.106
\end{tabular}

function. $36.7 \%$ of study participants revealed the presence of pulmonary fibrosis.

The HRT parameters were classified in accordance with current standards [13]. Among patients with SSC 19 had at least 5 ventricular beats suitable for analysis. Abnormal values of To $\geq 0 \%$ and/or Ts $\leq 2.5 \mathrm{~ms} / \mathrm{RR}$ occurred only in this group. One person had two abnormal parameters $-T_{0}=0.63 \%$ and $T s=2.2 \mathrm{~ms} / \mathrm{RR}$ and only one abnormal To $=2.58 \mathrm{~ms} / \mathrm{RR}$. All the control group had correct To and Ts parameters. The median values were To $=-1.24 \%$ ( $\min .-10.71 ; \max .2 .58)$ and $T s=11.13 \mathrm{~ms} / \mathrm{RR}$ (min. 2.20; max. 28.65). These values did not differ significantly as compared with the control group, where the median values were $T_{0}=-1.36 \%$ ( $\min .-4.53$, $\left.\max .-0.41\right)$, and $T s=11.46 \mathrm{~ms} / \mathrm{RR}$ ( $\min .2 .60 ; \max .31 .80$ ).

Statistically significant differences were noted in the majority of evaluated HRV parameters between women with SSc and the control group (Table 2). These were lower in the case of SDNN, SDNN-ix and SDANN and higher for $\mathrm{p} 50 \mathrm{NN}$. In turn, the frequency domain parameters were generally lower in SSc patients.

There were no differences in any of the evaluated parameters depending on the menopausal status in both groups.

Table 2. Comparison of HRV parameters in time and frequency domains between the study and the control group in a 24-hour period indicates significant $(p<0.05)$ differences between the sclerotic and control group

\begin{tabular}{lccc}
\hline Parameter & SCL $(n=31)$ & Control $(n=30)$ & $P$-value \\
\hline Mean NN [ms] & $797.7 \pm 81.4$ & $838.3 \pm 89.2$ & 0.096 \\
\hline SDNN [ms] & $136.1 \pm 39.9$ & $156.6 \pm 29.8$ & 0.024 \\
\hline SDNN-ix [ms] & $41.0 \pm 12.2$ & $48.5 \pm 11.7$ & 0.031 \\
\hline SDANN [ms] & $122.9 \pm 37.8$ & $143.2 \pm 29.5$ & 0.017 \\
\hline r-MSSD [ms] & $34.0 \pm 20.5$ & $35.7 \pm 16.3$ & 0.264 \\
\hline p50NN (\%) & $11.2 \pm 26.4$ & $10.5 \pm 7.8$ & 0.035 \\
\hline Trl & $75.9 \pm 20.0$ & $81.8 \pm 16.7$ & 0.223 \\
\hline TINN & $694.7 \pm 237.3$ & $726.8 \pm 107.4$ & 0.283 \\
\hline TP [ms $\left.{ }^{2}\right]$ & $11845.3 \pm 6029.8$ & $12746.2 \pm 4289.9$ & 0.093 \\
\hline HF [ms $\left.{ }^{2}\right]$ & $3372.90 \pm 2535.19$ & $3762.67 \pm 1677.68$ & 0.082 \\
\hline LF [ms $\left.{ }^{2}\right]$ & $2894.5 \pm 1673.8$ & $3379.4 \pm 1060.7$ & 0.011 \\
\hline VLF $\left[\mathrm{ms}^{2}\right]$ & $2853.5 \pm 871.7$ & $3450.6 \pm 787.6$ & 0.003 \\
\hline ULF [ms $\left.{ }^{2}\right]$ & $854.4 \pm 211.7$ & $1002.4 \pm 211.7$ & 0.018 \\
\hline NHF & $39.7 \pm 5.4$ & $42.7 \pm 5.3$ & 0.035 \\
\hline NLF & $36.8 \pm 6.5$ & $40.2 \pm 5.3$ & 0.025 \\
\hline LF/HF & $0.95 \pm 0.26$ & $0.96 \pm 0.23$ & 0.746 \\
\hline & & & \\
\hline
\end{tabular}


Table 3. Comparison of HRV parameters in time and frequency domains between the study and the control group in 4-hour-long periods (morning activity hours 8:00-12:00; night resting hours 00:00-4:00); indicates significant $(p<0.05)$ differences between the sclerotic and the control group

\begin{tabular}{|c|c|c|c|}
\hline Parameter & $\begin{array}{c}\mathrm{SCL} \\
(n=31)\end{array}$ & $\begin{array}{l}\text { Control } \\
(n=30)\end{array}$ & $P$-value \\
\hline \multicolumn{4}{|c|}{ Morning activity hours: } \\
\hline Mean NN [ms] & $724.2 \pm 95.0$ & $785.4 \pm 78.4$ & 0.003 \\
\hline SDNN [ms] & $105.7 \pm 27.9$ & $125.4 \pm 44.0$ & 0.082 \\
\hline SDNN-ix [ms] & $35.9 \pm 9.9$ & $44.4 \pm 11.3$ & 0.003 \\
\hline SDANN [ms] & $95.8 \pm 27.0$ & $114.0 \pm 43.0$ & 0.087 \\
\hline $\mathrm{r}-\mathrm{MSSD}[\mathrm{ms}]$ & $27.3 \pm 15.0$ & $30.8 \pm 13.1$ & 0.113 \\
\hline p50NN (\%) & $4.9 \pm 6.2$ & $7.4 \pm 5.8$ & 0.019 \\
\hline $\mathrm{Trl}$ & $49.7 \pm 13.4$ & $56.5 \pm 19.0$ & 0.334 \\
\hline TINN & $513.6 \pm 184.4$ & $528.6 \pm 282.3$ & 0.724 \\
\hline $\mathrm{TP}\left[\mathrm{ms}^{2}\right]$ & $4090.5 \pm 1592.4$ & $4858.5 \pm 1615.6$ & 0.031 \\
\hline $\mathrm{HF}\left[\mathrm{ms}^{2}\right]$ & $1096.5 \pm 727.2$ & $1318.3 \pm 677.0$ & 0.075 \\
\hline $\operatorname{LF}\left[\mathrm{ms}^{2}\right]$ & $1013.4 \pm 426.2$ & $1276.5 \pm 450.6$ & 0.012 \\
\hline $\mathrm{VLF}\left[\mathrm{ms}^{2}\right]$ & $1050.0 \pm 255.8$ & $1331.4 \pm 390.0$ & 0.003 \\
\hline $\mathrm{ULF}\left[\mathrm{ms}^{2}\right]$ & $424.5 \pm 116.4$ & $505.6 \pm 155.9$ & 0.082 \\
\hline $\mathrm{NHF}$ & $40.1 \pm 5.2$ & $41.8 \pm 5.6$ & 0.135 \\
\hline NLF & $40.6 \pm 7.9$ & $43.0 \pm 6.1$ & 0.360 \\
\hline $\mathrm{LF} / \mathrm{HF}$ & $1.0 \pm 0.3$ & $1.1 \pm 0.3$ & 0.874 \\
\hline \multicolumn{4}{|l|}{ Night resting hours: } \\
\hline Mean NN [ms] & $907.7 \pm 111.5$ & $986.3 \pm 108.1$ & 0.012 \\
\hline SDNN [ms] & $74.4 \pm 28.7$ & $98.2 \pm 27.7$ & 0.002 \\
\hline SDNN-ix [ms] & $45.1 \pm 16.7$ & $59.8 \pm 18.9$ & $<0.001$ \\
\hline SDANN [ms] & $58.2 \pm 20.8$ & $74.1 \pm 18.6$ & 0.002 \\
\hline r-MSSD [ms] & $34.8 \pm 23.5$ & $47.7 \pm 22.7$ & 0.009 \\
\hline p50NN (\%) & $7.6 \pm 9.5$ & $19.0 \pm 14.4$ & $<0.001$ \\
\hline Trl & $35.3 \pm 15.8$ & $46.8 \pm 14.5$ & 0.001 \\
\hline TINN & $293.3 \pm 140.8$ & $401.9 \pm 150.4$ & 0.002 \\
\hline $\mathrm{TP}\left[\mathrm{ms}^{2}\right]$ & $4344.0 \pm 2359.4$ & $5685.4 \pm 2085.8$ & 0.006 \\
\hline $\mathrm{HF}\left[\mathrm{ms}^{2}\right]$ & $1377.9 \pm 1045.3$ & $1866.5 \pm 872.1$ & 0.008 \\
\hline $\mathrm{LF}\left[\mathrm{ms}^{2}\right]$ & $1189.7 \pm 740.5$ & $1658.3 \pm 733.8$ & 0.007 \\
\hline $\mathrm{VLF}\left[\mathrm{ms}^{2}\right]$ & $1060.6 \pm 359.8$ & $1412.8 \pm 412.3$ & 0.001 \\
\hline $\mathrm{ULF}\left[\mathrm{ms}^{2}\right]$ & $275.9 \pm 91.3$ & $348.4 \pm 111.6$ & 0.015 \\
\hline $\mathrm{NHF}$ & $44.78 \pm 7.1$ & $46.7 \pm 5.5$ & 0.234 \\
\hline NLF & $40.7 \pm 7.8$ & $42.7 \pm 6.3$ & 0.337 \\
\hline $\mathrm{LF} / \mathrm{HF}$ & $1.0 \pm 0.3$ & $0.9 \pm 0.3$ & 0.751 \\
\hline
\end{tabular}

A separate analysis for the morning activity hours and for night resting hours are shown in Table 3. In the morning activity, SSc patients had significantly lower proved average NN, SDNN-ix, p5ONN, TP, LF and VLF. In the bedtime, in SSc group all of the time domain, and
Table 4. Comparison of HRV parameters in time and frequency domains between morning activity hours and night resting hours in the study and control groups in 4-hour-long periods (morning activity hours 8:00-12:00; night resting hours 00:00-4:00); significant $(p<0.05)$ differences between the sclerotic and the control group

\begin{tabular}{|c|c|c|c|}
\hline Parameter & $\begin{array}{l}\text { Morning activity } \\
\text { hours }\end{array}$ & $\begin{array}{l}\text { Night resting } \\
\text { hours }\end{array}$ & $P$-value \\
\hline \multicolumn{4}{|l|}{ Sclerosis $(n=31)$ : } \\
\hline Mean NN [ms] & $724.2 \pm 95.0$ & $907.7 \pm 111.5$ & $<0.001$ \\
\hline SDNN [ms] & $105.7 \pm 27.9$ & $74.4 \pm 28.7$ & $<0.001$ \\
\hline SDNN-ix [ms] & $35.9 \pm 9.9$ & $45.1 \pm 16.7$ & 0.024 \\
\hline SDANN [ms] & $95.8 \pm 27.0$ & $58.2 \pm 20.8$ & $<0.001$ \\
\hline $\mathrm{r}-\mathrm{MSSD}[\mathrm{ms}]$ & $27.3 \pm 15.0$ & $34.8 \pm 23.5$ & 0.190 \\
\hline p50NN (\%) & $4.9 \pm 6.2$ & $7.6 \pm 9.5$ & 0.368 \\
\hline $\mathrm{Trl}$ & $49.7 \pm 13.4$ & $35.3 \pm 15.8$ & $<0.001$ \\
\hline TINN & $513.6 \pm 184.4$ & $293.3 \pm 140.8$ & $<0.001$ \\
\hline $\mathrm{TP}\left[\mathrm{ms}^{2}\right]$ & $4090.5 \pm 1592.4$ & $4344.0 \pm 2359.4$ & 0.822 \\
\hline $\mathrm{HF}\left[\mathrm{ms}^{2}\right]$ & $1096.5 \pm 727.2$ & $1377.9 \pm 1045.3$ & 0.254 \\
\hline $\operatorname{LF}\left[\mathrm{ms}^{2}\right]$ & $1013.4 \pm 426.2$ & $1189.7 \pm 740.5$ & 0.499 \\
\hline $\mathrm{VLF}\left[\mathrm{ms}^{2}\right]$ & $1050.0 \pm 255.8$ & $1060.6 \pm 359.8$ & 0.899 \\
\hline $\mathrm{ULF}\left[\mathrm{ms}^{2}\right]$ & $424.5 \pm 116.4$ & $275.9 \pm 91.3$ & $<0.001$ \\
\hline $\mathrm{NHF}$ & $40.1 \pm 5.2$ & $44.7 \pm 7.1$ & 0.011 \\
\hline NLF & $40.6 \pm 7.9$ & $40.7 \pm 7.8$ & 0.961 \\
\hline $\mathrm{LF} / \mathrm{HF}$ & $1.0 \pm 0.3$ & $1.0 \pm 0.3$ & 0.246 \\
\hline \multicolumn{4}{|l|}{ Control $(n=30)$ : } \\
\hline Mean NN [ms] & $785.4 \pm 78.4$ & $986.3 \pm 108.1$ & $<0.001$ \\
\hline SDNN [ms] & $125.4 \pm 44.0$ & $98.2 \pm 27.7$ & 0.011 \\
\hline SDNN-ix [ms] & $44.4 \pm 11.3$ & $59.8 \pm 18.9$ & $<0.001$ \\
\hline SDANN [ms] & $114.0 \pm 43.0$ & $74.1 \pm 18.6$ & $<0.001$ \\
\hline $\mathrm{r}-\mathrm{MSSD}$ [ms] & $30.8 \pm 13.1$ & $47.7 \pm 22.7$ & 0.002 \\
\hline p50NN [\%] & $7.4 \pm 5.8$ & $19.0 \pm 14.4$ & $<0.001$ \\
\hline Trl & $56.5 \pm 19.0$ & $46.8 \pm 14.5$ & 0.022 \\
\hline TINN & $528.6 \pm 282.3$ & $401.9 \pm 150.4$ & 0.045 \\
\hline $\mathrm{TP}\left[\mathrm{ms}^{2}\right]$ & $4858.5 \pm 1615.6$ & $5685.4 \pm 2085.8$ & 0.158 \\
\hline $\mathrm{HF}\left[\mathrm{ms}^{2}\right]$ & $1318.3 \pm 677.0$ & $1866.5 \pm 872.1$ & 0.017 \\
\hline $\mathrm{LF}\left[\mathrm{ms}^{2}\right]$ & $1276.5 \pm 450.6$ & $1658.3 \pm 733.8$ & 0.044 \\
\hline $\mathrm{VLF}\left[\mathrm{ms}^{2}\right]$ & $1331.4 \pm 390.0$ & $1412.8 \pm 412.3$ & 0.605 \\
\hline $\mathrm{ULF}\left[\mathrm{ms}^{2}\right]$ & $505.6 \pm 155.9$ & $348.4 \pm 111.6$ & $<0.001$ \\
\hline $\mathrm{NHF}$ & $41.8 \pm 5.6$ & $46.7 \pm 5.5$ & 0.003 \\
\hline $\mathrm{NLF}$ & $43.0 \pm 6.1$ & $42.7 \pm 6.3$ & 0.982 \\
\hline $\mathrm{LF} / \mathrm{HF}$ & $1.1 \pm 0.3$ & $0.9 \pm 0.3$ & 0.057 \\
\hline
\end{tabular}

most frequency domain parameters were statistically significantly lower compared to the healthy group.

Next, circadian variations of HRV were evaluated (Table 4). In the control group all of time domain parameters were significantly different between the morning activity 
and night resting hours. Also among the parameters of frequency domain there were significant differences for HF, LF, ULF and NHF noted. In women with SSc, there were no such differences for r-MSSD and p50NN. In turn, of the frequency domain parameters such differences occurred only for ULF and NHF.

It revealed the presence of a statistically significant $(p<0.05)$ positive correlation between To and Ix-Triangle $(R=0.463)$, and a negative correlation between To and $\operatorname{NLF}(R=-0.666)$, To and LF/HF ratio $(R=-0.468)$, Ts and IX-Triangle $(R=-0.456)$ and between TS and TINN $(R=$ -0.467). In addition, there was a negative correlation between the duration of the disease and To $(R=-0.530)$ and between waist circumference and Ts $(R=-0.471)$ and a positive correlation between the body weight and To $(R=0.468)$.

\section{Discussion}

Cardiac involvement including arrhythmia and conductivity disturbance, have an important prognostic value in patients with SSc [1, 4, 8, 23-26]. The main causes of cardiac abnormalities include the autonomic disorders, vasomotor disturbances on the small coronary vessels, including vascular endothelium and progressive fibrosis [27-30]. The early symptoms of cardiac involvement are very uncharacteristic, however, approx. $5 \%$ of deaths are sudden [31-33]. Simple and non-invasive method of assessment of the cardiovascular risk and function of the autonomic nervous system is to estimate the HRT and HRV parameters. Among them SDANN and LF the best reflect the function of the sympathetic, r-MMSD, p50NN and $\mathrm{HF}$ - parasympathetic nervous function [12, 34, 35]. Low values of SDNN are furthermore associated with a higher risk of developing arterial hypertension and progression of atherosclerotic lesions [36]. The HRT is a reflection of the total sympathetic-parasympathetic balance, and also reflects baroreflex sensitivity [12, 14].

In our study we found significantly lower SDANN and LF values in patients with SSc, which reflects an increase in sympathetic activities. In turn, parameters of parasympathetic activity did not change significantly, or in case of the value p50NN slightly, but are statistically significantly increased. These results are consistent with observations of Di Franco et al. [9], who showed significantly lower values of SDNN, SDANN and p50NN, indicators of overexpression of the sympathetic nervous system and the lack, or only slight impairment, of parasympathetic activity. The authors also suggest that sympathetic hyperactivity might disturb the balance between vasoconstriction and vasodilatation in favour of the first one. In contrast to these results, Suarez-Almazor et al. [37] do not confirm cardiovascular autonomic insufficiency in SSc patients and CREST syndrome, but they used other available methods, rather than HRT and HRV. Time and frequency domain parameters were significantly lower compared with the control group in Othman et al. study [38]. The authors found no significant correlation between HRV parameters and the subtype and duration of the disease. This is consistent with our observations: we found no significant correlations between the HRV parameters and time after diagnosis. Othman puts an interesting hypothesis: changes in the HRV and arrhythmias may occur at a very early, asymptomatic stage of SSc, even when there is no change in the cardiovascular system detected by routine diagnostic methods. Similar observations were obtained by Sielańczyk et al. [17]. They found no statistically significant difference in HRV parameters between the two groups of subjects with SSc duration of less than 8 and more than 10 years. Bienias et al. [18], using HRT and HRV parameters, also showed dominance of the sympathetic nervous system in SSc patients. However, the average duration of the disease was 11.7 years in this case. It can therefore be assumed that the subjects were in a more advanced stage of the disease. No differences depending on the subtype of the disease were found. Deterioration of HRT with the duration of illness was found. These authors suggest that the autonomic nervous system disorders in patients with SSc assessed by HRT and $H R V$, increased with the progress of the disease. This was not confirmed by the results of our studies as there was no correlation between HRT and HRV parameters and duration of SSc. Furthermore, HRT parameters did not differ significantly as compared with the control group. It is possible that changes of HRT may appear only in the later stages, as opposed to aberrant HRV. Abnormal HRT correlated with the escalation of arrhythmia, which is characteristic in the overt involvement of the cardiovascular system. In the study of Bienias et al. [22], a decrease in the value of Ts was associated with a higher incidence of ventricular arrhythmias. The frequency of this type of arrhythmia, including nsVT, increases with the duration and severity of lesions. As it is known, abnormal HRT parameters are the reflection of a greater risk of death, especially sudden deaths, associated with serious ventricular arrhythmias. Such a clear relationship was demonstrated for many diseases and clinical conditions [12, 14, 16, 39, 40]. Bienias et al. showed that among SSc patients most people with HP are characterized by abnormal To and/or Ts values. The presence of HP was itself an independent factor affecting the HRT disturbances. It seems that the appearance of abnormal HRT values is a strong risk factor for sudden cardiac death. In our observation, only two people were in the risk group.

Another problem is the loss of diurnal variation of the autonomic nervous system, which is associated with a higher cardiovascular risk. In the general population, a normal circadian rhythm of HRV occurs, with predominantly sympathetic nervous system during the day and parasympathetic at night. In our study, at night there was a significant decrease in the values of SDANN, r-MMD and $\mathrm{p} 50 \mathrm{NN}$ as well as HF. There was an indicator of sig- 
nificant dominance of the sympathetic nervous system and decreasing the activity of the parasympathetic nervous system. A similar issue was highlighted by Di Franco et al. [9] on the basis of reduced SDNN and SDANNindex. Similar findings were observed for patients with diabetes, as well as in the offspring of diabetic patients who are not yet diagnosed with diabetes [41]. It is characterized by the lack of the increase in the value of SDNN and r-MMSD and p50NN [34]. Similarly, this is the case of decompensated liver cirrhosis [42]. The loss of diurnal variation HRV appears in the early stages of SSc.

The study has its limitations. It was performed on a relatively small, but homogeneous population. We used data available only in the female population. There are reports that menopause alters autonomic balance. Moodithaya et al. [43] have shown a decrease in HF and LF values in postmenopausal women. However, these authors indicate the key effect of age on the parameters of autonomic function. Similarly, Tezini et al. [44] indicate a lack of correlation between the autonomic function and ovarian hormone deprivation in the case of natural menopause. In turn, Neufeld et al. [45], despite showing a significant difference in HF and LF values, do not find a clinically important relationship between cardiovascular autonomic control and menopausal status in women. So, despite a slightly higher number of postmenopausal women in our study, we decided that this fact did not have a significant impact on the results we obtained. Authors have tried to minimize the impact of other factors beyond the SSc, including co-morbidities and medications on HRT and HRV. However, it seems that the results are promising and could become a contribution to further investigations.

\section{Conclusions}

Cardiovascular system involvement is referred to as Scleroderma Heart Disease (SHD) by Ferri et al. [8]. The study confirmed the presence of HRV disturbances, including HRV circadian rhythm, as it may seem at an early stage of SSc. HRT disorders may be characterized by the increasing changes with advancing disease. This indicates the presence of autonomic imbalance and the increased cardiovascular risk. Use of the 24-hour ECG Holter monitoring with HRV and HRT appears to be completely safe and inexpensive. This requires further studies.

\section{Acknowledgments}

Poliwczak A.R. and Dworniak K. are the co-first authors of the manuscript.

Publication co-financed by the European Social Fund and the State Budget under Measure 2.6 of the Integrated Operational Programme for Regional Development, in connection with the implementation of the project called "Grants supporting innovative research of postgraduates" and statutory funds 503/1-152-01/503-11-002.

\section{Conflict of interest}

The authors declare no conflict of interest.

\section{References}

1. Mitchell H, Bolster MB, Le Roy EC. Scleroderma and related conditions. Med Clin North Am 1997; 81: 129-49.

2. Abraham DJ, Krieg T, Distler J, Distler O. Overview of pathogenesis of systemic sclerosis. Rheumatology 2009; 48 Suppl: ii3-7.

3. Steen V. The heart in systemic sclerosis. Curr Rheumatol Rep 2004; 6: 137-40.

4. Kahan A, Coghlam G, McLaughlin V. Cardiac complications of systemic sclerosis. Rheumatology 2009; 48 Suppl: i45-8.

5. Ioannidis JPA, Vlachoyiannopoulos PG, Haidich AB, et al. Mortality in systemic sclerosis: an international meta-analysis of individual patient data. Am J Med 2005; 118: 2-10.

6. Herrick LA. Pathogenesis of Renaud's phenomenon. Rheumatology 2005; 44: 587-96.

7. Tzelepis GE, Kelekis NL, Plastiras SC, et al. Pattern and distribution of myocardial fibrosis in systemic sclerosis. Arthritis Rheumatism 2007; 56: 3827-36.

8. Ferri C, Emdin M, Giuggioli D, et al. Autonomic dysfunction in systemic sclerosis: time and frequency domain 24 hour heart variability analysis. Br J Rheumatol 1997; 36: 669-76.

9. Di Franco M, Paradiso M, Ricci V, et al. Autonomic dysfunction and microvascular damage in systemic sclerosis. Clin Rheumatol 2007; 26: 1278-83.

10. Ciftci O, Onat AM, Yavuz B, et al. Cardiac repolarisation abnormalities and increased sympathetic activity in scleroderma. J Natl Med Assoc 2007; 99: 232-7.

11. Iwasa A, Hwa M, Hassankhani A, et al. Abnormal heart turbulence predicts the initiation of ventricular arrhythmias. PACE 2005; 28: 1189-97.

12. Task Force of the European Society of Cardiology and North American Society of Pacing and Electrophisiology. Heart rate variability: standards of mesurement, physiologgical interpretation and clinical use. Circulation 1996; 93: 1043-65.

13. Bauer A, Malik A, Schmidt G, et al. Heart rate turbulence: standards of measurement, physiological interpretation, and clinical use. JACC 2008; 52: 1353-65.

14. Schmidt G, Malik M, Barthel P, et al. Heart-rate turbulence after ventricular premature beats as a predictor of mortality after acute myocardial infarction. Lancet 1999; 353: 1390-6.

15. Wichterle D, Simek J, La Rovere MT, et al. Prevalent low-frequency oscillation of heart rate. new predictor of mortality after myocardial infarction. Circulation 2004; 110: 1183-90.

16. Poliwczak AR, Białkowska J, Broncel M, et al. Heart rhythm turbulence and NT-proBNP in decompensated liver cirrhosis - a pilot study. Med Sci Monit 2011; 17: 5-11.

17. Sielańczyk AW, Jagodziński L, Gmyrek J, et al. Heart rate variability in patients with systemic sclerosis. Acta Medica Lituanica 2007; 14: 46-9.

18. Bienias P, Ciurzyński M, Glińska-Wielochowska M, et al. Heart rate turbulence assessment in systemic sclerosis: the role for the detection of cardiac autonomic nervous dysfunction. Rheumatology 2010; 49: 355-60.

19. Masi AT, Medsger TA, Rodnam GP, et al. Preliminary criteria for the classification of systemic sclerosis (scleroderma). Arthritis Rheum 1980; 23: 581-90.

20.Schneider R, Barthel P, Watanabe M. Heart rate turbulence on Holter. In: Dynamic Electrocardiography. Malik M, Camm JA (eds.). Blackwell Futura Publishing, London 2004; 190-3. 
21. Watanabe MA, Schmidt G. Heart rate turbulence: a 5-year revieve. Heart Rhythm 2004; 1: 732-8.

22. Bienias P, Ciurzyński M, Glińska M, et al. Heart rate turbulence impairment and ventricular arrhythmias in patients with systemic sclerosis. PACE 2010; 33: 920-8.

23. Vlachoyiannopoulos PG, Dafni UG, Pakas I, et al. Systemic scleroderma in Greece: low mortality and strong linkage with HLA-DRB1*1104 allele. Ann Rheum Dis 2000; 59: 359-67.

24. Steen VD, Medsger TA Jr. Severe organ involvement in systemic sclerosis with diffuse scleroderma. Arthritis Rheum 2000; 43: 2437-44.

25. Ferri C, Valentini G, Cozzi F, et al. Systemic sclerosis: demographic, clinical, and serologic features and survival in 1,012 Italian patients. Medicine (Baltimore) 2002; 81: 139-53.

26. Clements PJ, Lachenbruch PA, Furst DE, et al. Cardiac score. A semiquantitative measure of cardiac involvement that improves prediction of prognosis in systemic sclerosis. Arthritis Rheum 1991; 34: 1371-80.

27. Follansbee WP, Curtiss El, Medsger TA Jr, et al. Physiologic abnormalities of cardiac function in progressive systemic sclerosis with diffuse scleroderma. N Engl J Med 1984; 310: 142-8.

28. Kahan A, Devaux JY, Amor B, et al. Nifedipine and thallium-201 myocardial perfusion in progressive systemic sclerosis. N Engl J Med 1986; 314: 1397-402.

29. Kahan A, Allanore Y. Primary myocardial involvement in systemic sclerosis. Rheumatology (Oxford) 2006; 45 Suppl: 14-7.

30. Hachulla AL, Launay D, Gaxotte V, et al. Cardiac magnetic resonance imaging in systemic sclerosis: a cross-sectional observational study of 52 patients. Ann Rheum Dis 2009; 68: 1878-84.

31. Follansbee WP, Zerbe TR, Medsger TA Jr. Cardiac and skeletal muscle disease in systemic sclerosis (scleroderma): a high risk association. Am Heart J 1993; 125: 194-203.

32. Steen VD, Medsger TA Jr. Changes in causes of death in systemic sclerosis, 1972-2002. Ann Rheum Dis 2007; 66: 940-4.

33. Di Bona GF, Kopp UC. Neural control of renal function. Physiol Rev 1997; 77: 75-197.

34. Fiorentini A, Perciaccante A, Paris A, et al. Circadian rhythm of autonomic activity in non diabetic offsprings of type 2 diabetic patients. Cardiovasc Diabetol 2005; 4: 15.

35. Stein PK. Assessing heart variability from real-world Holter reports. Card Electrophysiol Rev 2002; 6: 239-44.

36. Dekker JM, Crow RS, Folson AR, et al. Low heart rate variability in a 2-minute rhythm strip predicts risk of coronary heart disease and mortality from several causes: the ARIC study. Circulation 2000; 102: 1239-44.

37. Suarez-Almazor ME, Bruera E, Russell AS. Normal cardiovascular autonomic function in patients with systemic sclerosis (CREST variant). Ann Rheum Dis 1988; 47: 672-4.

38. Othman KM, Assaf NY, Farouk HM, Aly Hassan IM. Autonomic dysfunction predicts early cardiac affection in patients with systemic sclerosis. Clin Med Insights Arthritis Musculoskelet Disord 2010; 3: 43-54.

39. Davies LC, Francis DP, Ponikowski P, et al. Relation of heart rate and blood pressure turbulence following premature ventricular complexes to baroreflex sensitivity in chronic congestive heart failure. Am J Cardiol 2001; 87: 737-42.

40.Bienias P, Ciurzyński M, Korczak D, et al. Pulmonary hypertension in systemic sclerosis determines cardiac autonomic dysfunction assessed by heart rate turbulence. Int I Cardiol 2010; 141: 322-5.
41. Kardelen F, Akçurin G, Ertuğ H, et al. Heart rate variability and circadian variations in type 1 diabetes mellitus. Pediatric Diabetes 2006; 7: 45-50.

42. Białkowska J, Poliwczak AR, Jabłkowski M, et al. Heart rate variability in patients with decompensated liver cirrhosis a pilot study. Prz Gastroenterol 2012; 7: 149-55.

43. Moodithaya SS, Avadhany ST. Comparison of cardiac autonomic activity between pre and post menopausal women using heart rate variability. Indian J Physiol Pharmacol 2009; 53: 227-34.

44. Tezini GC, Becari C, Zanotto CZ, et al. Ageing is the main determinant of haemodynamics and autonomic cardiac changes observed in post-menopausal female rats. Auton Neurosci 2013; 174: 36-41.

45. Neufeld IW, Kiselev AR, Karavaev AS, et al. Autonomic control of cardiovascular system in pre- and postmenopausal women: a cross-sectional study. J Turk Ger Gynecol Assoc 2015; 16: 11-20. 